\section{Solar terrestrial physics}

\section{Global geospace study}

\author{
from M.J. Rycroft, A.J. Smith, D. Jones, J.R. Dudeney and S.D. Shawhan
}

A MAJOR international programme to study 'geospace' is currently being planned by scientists in the USA, Japan and Europe. The term geospace was coined recently to describe the ensemble of separate regions close to the Earth and traditionally called the atmosphere, ionosphere, magnetosphere, magnetosheath and solar wind. The single word emphasizes that although these systems have until now been explored separately - for example, the magnetosphere in the International Magnetospheric Study (1976-79) and the stratosphere and mesosphere in the Middle Atmosphere Programme (1982-85) - they are in fact intimately coupled through a wide variety of mass, momentum and energy transfer processes and the time has now come to study them as a whole.

The Global Geospace Study (GGS) has two main scientific aims: to understand how energy from the Sun, particularly that carried by the solar wind and impinging on geospace, is transferred into, and converted by, the geospace system until it is eventually dissipated as heat in the ionosphere and upper atmosphere; and to understand the origin, transport and energy-gaining and -loss processes of the plasma populations of geospace. These questions are important for two basic reasons. First, they give insight into fundamental plasma physics. Some 99 per cent of matter in the Universe exists as plasma. The behaviour of cosmic plasma is not easily simulated in the laboratory, however, and geospace contains a vast natural plasma readily accessible both to detailed measurements in situ and to remote-sensing studies. The second reason is that an improved understanding of the response of geospace to solar variation has practical applications in radio communications, distribution of electrical power, geomagnetic surveys for minerals and hydrocarbons (particularly at high latitudes), space applications engineering and, possibly, the weather.

If the plans are approved, four spacecraft will be launched in the early 1990 s to make concerted and comprehensive observations of four key components of geospace (see the figure). The spacecraft, known as Wind and Polar, will focus on the two sources of flowing plasma, in the solar wind and in the ionosphere and magnetosphere (particularly the polar regions), while the Equator and Geotail spacecraft will concentrate on the two principal energy-storage regions, the ring current and the geomagnetic tail. Extensive observations made from the ground using modern techniques and a strong theoretical and modelling effort complete the six components of GGS.

All the spacecraft will make plasma, par- ticle and field measurements, though the exact specifications of their instruments (for example, energy range, sensitivity, frequency range) will be tailored to suit the particular region being studied. The spacecraft Wind, orbiting on the sunward side of geospace, will measure temporal changes of plasma density and velocity, ion composition, temperature and magnetic field strength in the solar wind input. The effects of these changes will then be measured on Geotail, Polar and Equator, and on the ground. For example, it is believed that a change in the interplanetary magnetic field embedded in the solar wind can trigger a 'substorm' in which energy stored in the geomagnetic tail is suddenly released into the auroral zones. This process might be observed by Geotail as a change in magnetic field direction and as plasma acceleration; the relative timing of these events will give information about the physical processes involved and their relation to one another. Increases in the energetic particle fluxes in the ring current and in plasma wave activity would be detected by Equator, enhanced auroral emissions in the polar regions by Polar, and ionospheric heating, electric currents, plasma flows and wave generation observed from the ground at high latitudes.

Three spacecraft (Wind, Equator and Polar) will be built by NASA and the fourth (Geotail) by the Institute of Space and Aeronautical Science, Japan. The instruments will be provided by US and Japanese principal investigators working closely with European and American coinvestigators. Four ground-based facilities have been proposed: the incoherent scatter radar at Sondrestromfjord, Greenland, a dual auroral radar network in the Arctic and a Canadian network of ground instruments for the Northern Hemisphere; and for the Southern Hemisphere, a comprehensive array of experiments at and around the British Antarctic Survey station, Halley $\left(76^{\circ} \mathrm{S}, 27^{\circ} \mathrm{W}\right.$ geographic).

In planning GGS, the importance of efficient and rapid handling, processing and dissemination of the data, using the most modern computer and communications technology available, has been recognized. Data from the spacecraft will be processed at a central data-handling facility before being sent to investigators, probably in the form of optical discs. Ground data will be inserted into the system in a similar way, the Antarctic data being transmitted from Antarctica via a geostationary satellite in order to be available rapidly.

GGS is a component of the even wider International Solar Terrestrial Programme, agreed by the space agencies of USA, Europe and Japan. Other space missions, which will concentrate on solar observations (Soho) and magnetospheric microphysics (Cluster) and will complement GGS, are currently being investigated by the European Space Agency.

M.J. Rycroft, A.J. Smith, D. Jones and J.R. Dudeney are at the British Antarctic Survey, Madingley Road, Cambridge CB3 OET, and S.D. Shawhan is at NASA Headquarters, Washington, DC 20546.

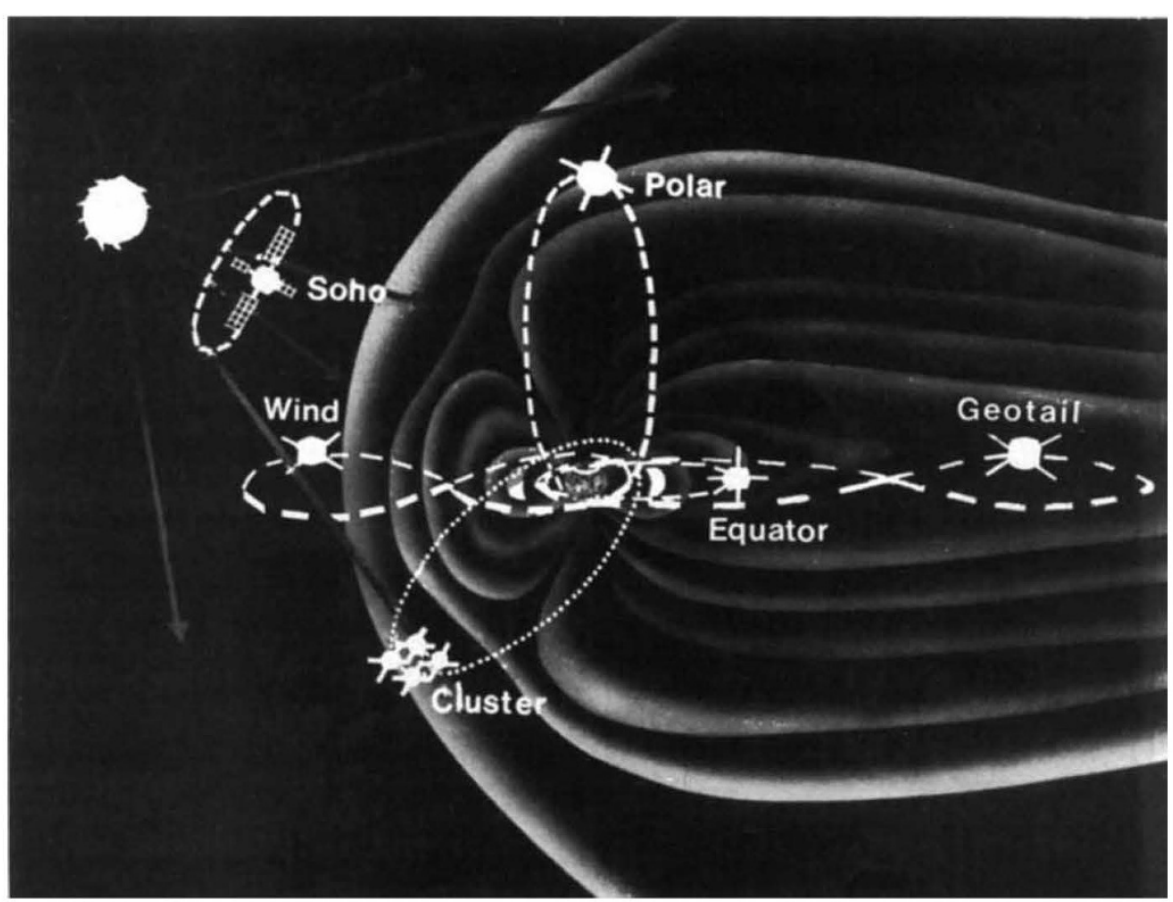

Targets for spacecraft of the International Solar Terrestrial Programme. Wind will focus on the solar wind, Polar on polar regions of the ionosphere and magnetosphere, Cluster on magnetospheric microphysics, Soho on the Sun, and Equator and Geotail on the two main energy-storage regions, the ring current and geomagnetic tail respectively. 